\title{
Crystal Structure and Local Dynamics in Tetrahedral Proton-Conducting $\mathrm{La}_{1-x} \mathrm{Ba}_{1+x} \mathrm{GaO}_{4}$
}

\author{
Francesco Giannici, ${ }^{* \dagger}{ }^{\dagger}$ Diego Messana, ${ }^{\dagger}$ Alessandro Longo, ${ }^{\ddagger}$ and Antonino Martorana ${ }^{\dagger}$ \\ Dipartimento di Chimica Inorganica e Analitica "Stanislao Cannizzaro", Università degli Studi di Palermo, \\ Viale delle Scienze, I-90128 Palermo, Italy, and Istituto per lo Studio dei Materiali Nanostrutturati, Consiglio \\ Nazionale delle Ricerche, Via Ugo La Malfa 153, I-90146 Palermo, Italy
}

Received: July 5, 2010; Revised Manuscript Received: November 17, 2010

$\mathrm{La}_{1-x} \mathrm{Ba}_{1+x} \mathrm{GaO}_{4-\delta}$ (LBG) compounds, based on unconnected $\mathrm{GaO}_{4}$ moieties, were recently proposed as proton conductors. Protonic defects in the lattice are inserted through self-doping with $\mathrm{Ba}^{2+}$, to create oxygen vacancies subsequently filled by hydroxyl ions. We present a combined structural analysis on self-doped LBG using $\mathrm{X}$-ray diffraction (XRD) and X-ray absorption (EXAFS): these results unravel the finer structural details on the short-range and long-range scales, and they are correlated with the dynamical properties of protonic conduction coming from vibrational spectroscopy. The structure of the $\mathrm{GaO}_{4}$ groups is independent of the oxide composition. On hydration, an array of short intertetrahedral hydrogen bonds is formed, producing a contraction of the $a$ axis. On the basis of thermogravimetric analysis, EXAFS, XRD and infrared spectroscopy (IR) results, we propose that the stiffness of the $\mathrm{GaO}_{4}$ tetrahedra hinders the intratetrahedral proton transfer, while the noticeable fraction of protons involved in strong hydrogen bonds limit the proton reorientational freedom.

\section{Introduction}

Ion-conducting oxides are among the most studied materials for new electrochemical applications, and especially for fuel cells, since they are stable at high temperatures, thereby reducing the need for expensive catalysts and increasing the balance-ofplant. In particular, oxides with high proton conduction are especially sought-after, since the low activation energy makes them useful for midtemperature applications $\left(300-500{ }^{\circ} \mathrm{C}\right)$. Trivalent-doped II-IV perovskites (e.g., barium cerates) were the first candidates for high-temperature protonic conduction, and they have been extensively investigated in the last 25 years. ${ }^{1}$ In recent years, the research on proton-conducting perovskites has focused on different preparation methods to optimize barium cerates and zirconates; alternatively, the last new perovskite (Ydoped barium zirconate) was proposed more than 10 years ago, so that it is worth also exploring other structure types to achieve a mature fuel cell technology based on proton conductingoxides. ${ }^{2}$ Doped $\mathrm{LaNbO}_{4}$ were recently proposed, with better chemical stability and lower grain boundary impedance than most perovskites. ${ }^{3}$ A common trait to most of such alternative electrolytes is the presence of isolated tetrahedral moieties, like $\left[\mathrm{SiO}_{4}\right]$ in apatite rare-earth silicates, $\left[\mathrm{SO}_{4}\right]$ and $\left[\mathrm{PO}_{4}\right]$ in cesium acid salts, $\left[\mathrm{GeS}_{x}(\mathrm{OH})_{4-x}\right]$ in thiohydroxogermanates, $\left[\mathrm{NbO}_{4}\right]$ and $\left[\mathrm{TaO}_{4}\right]$ in lanthanum niobates and tantalates. ${ }^{2-5} \mathrm{~A}$ review of the solid-state chemistry that controls the performance of these new materials for electrolytes and electrodes has appeared very recently. ${ }^{6}$

The structure of $\mathrm{LaBaGaO}_{4}$ (LBG) (space group $P 2_{1} 2_{1} 2_{l}$ ) is based on a loose framework of $\mathrm{GaO}_{4}$ tetrahedra, separated by the large $\mathrm{La}^{3+}$ and $\mathrm{Ba}^{2+}$ cations. The protonic defects are introduced by doping the $\mathrm{La}^{3+}$ site with excess $\mathrm{Ba}^{2+}$ (single-

\footnotetext{
* To whom correspondence should be addressed. E-mail: giannici@ pa.ismn.cnr.it.

† Dipartimento di Chimica Inorganica e Analitica "Stanislao Cannizzaro", Università degli Studi di Palermo.

* Istituto per lo Studio dei Materiali Nanostrutturati, Consiglio Nazionale delle Ricerche.
}

phase solid solutions are formed up to $20 \%$ of Ba excess). To maintain charge neutrality, oxygen vacancies are created, and then filled by reaction with moisture to form hydroxyl defects:

$$
\begin{gathered}
2 \mathrm{BaO}+2 \mathrm{La}_{\mathrm{La}}^{x}+\mathrm{O}_{\mathrm{o}}^{x} \rightarrow 2 \mathrm{Ba}_{\mathrm{La}}^{\prime}+\mathrm{Vo}^{\bullet \bullet}+\mathrm{La}_{2} \mathrm{O}_{3} \\
\mathrm{Vo} \ddot{\bullet}+\mathrm{O}_{\mathrm{o}}^{x}+\mathrm{H}_{2} \mathrm{O}_{(\mathrm{g})} \rightarrow 2 \mathrm{OH}_{\mathrm{O}}^{\cdot} \\
\mathrm{La}_{1-x} \mathrm{Ba}_{1+x} \mathrm{GaO}_{4-x / 2}+x / 2 \mathrm{H}_{2} \mathrm{O}_{(\mathrm{g})} \rightarrow \mathrm{La}_{1-x} \mathrm{Ba}_{1+x} \mathrm{GaO}_{4} \mathrm{H}_{x}
\end{gathered}
$$

Upon heating over $500-700{ }^{\circ} \mathrm{C}$, or heat treatment in dry atmosphere, water is released from the lattice, giving rise to appreciable oxygen vacancy conduction. ${ }^{7}$ Proton and vacancy conduction in LBG has recently been investigated in a combined computational and neutron diffraction study. ${ }^{8}$ In that work, the calculated intratetrahedral transfer for protons was found to be rate-limiting, while the intertetrahedral hopping showed much lower activation barriers $(0.7$ vs $0.07 \mathrm{eV})$, ascribed to the formation of hydrogen bonds between neighboring $\mathrm{GaO}_{4}$ units. In the same work, it was proposed that in dry LBG compounds each oxygen vacancy is stabilized by the dimerization of two tetrahedra, which share a corner to form a $\mathrm{Ga}_{2} \mathrm{O}_{7}$ dimer, and that the oxygen vacancies can diffuse thanks to the dynamic formation and breaking of such dimers. In electrolytes like $\mathrm{CsHSO}_{4}$, based on tetrahedral sulfate units, the proton conduction undergoes a dramatic enhancement when a very symmetric "plastic phase" is formed, as a result of the rotational motion of the tetrahedra. ${ }^{9}$ Although there is no sharp transition or formation of superprotonic phase in LBG, the factors that control the structure-property relations in tetrahedra-based electrolytes are likely similar. In particular, the critical point to be addressed in LBG compounds and other high temperature proton conducting oxides with analogous structure is the reorientation ability 
of the tetrahedra to create transient hydrogen bonds that facilitate the proton hopping. ${ }^{8}$

In the past few years, we have successfully applied extended X-ray absorption fine structure (EXAFS) analysis to a variety of proton-conducting perovskites. ${ }^{10-15}$ In such cases, the local environment approach is ideally suited to elucidate the fine structural and dynamical details around a single cation. In the present work, which completes a preliminary brief report using different data and models, ${ }^{16}$ we apply EXAFS analysis to solve the structure of LBG up to about $4 \AA$ around $\mathrm{Ga}^{3+}$ : the results are correlated with high-resolution X-ray diffraction (HR-XRD) which also provides very good sensitivity to the oxygen sublattice due to the high quality of the data. Apart from site selectivity, it is worth remarking that these techniques are complementary in two respects: (a) XRD is mostly sensitive to the crystalline domains and has low sensitivity for grain boundaries and segregated phases, while EXAFS probes all of the absorber atoms (in this case, $\mathrm{Ga}$ ), regardless of their location; (b) the disorder factors probed by XRD are proportional to the displacement of the atoms from their lattice sites, while the disorder factors probed by EXAFS are proportional to the spread of an absorber-backscatterer bond. Therefore, if the motion of an absorber-backscatterer pair is correlated, then it results in very low disorder in the corresponding EXAFS signal, and high disorder in the XRD signal.

The static structural characterization is then coupled with the analysis of dynamics and electrical performance. The uptake and release of water, studied by thermogravimetry, is accompanied by structural transformations that are investigated with in situ XRD in a wide range of temperatures. Infrared spectroscopy (IR) is used to study the $\mathrm{O}-\mathrm{H}$ stretching region, and to investigate the hydrogen-bonding states of protons. The proton and oxygen-vacancy conductivity is measured using impedance spectroscopy.

These results eventually lead to a structural and functional understanding of LBG compounds and their transformations. The approach of the LBG structural analysis in relation with the proton conductivity points out general issues that can be taken into consideration for a better understanding of proton conductors based on unconnected tetrahedral units.

\section{Experimental Section}

Powders of nominal composition $\mathrm{La}_{1-x} \mathrm{Ba}_{1+x} \mathrm{GaO}_{4-\delta}$, with $x$ between 0 and 0.2 , were synthesized by solid-state route from $\mathrm{La}_{2} \mathrm{O}_{3}, \mathrm{Ga}_{2} \mathrm{O}_{3}$, and $\mathrm{BaCO}_{3}$, performing two calcinations at 1300 ${ }^{\circ} \mathrm{C}$ with intermediate grinding. Calcination at lower temperatures was not sufficient to achieve pure phase products and resulted in a mixture of $\mathrm{LaGaO}_{3}$ and $\mathrm{Ba}_{2} \mathrm{GaO}_{4}$. The powders were hydrated by equilibration with water vapor at $300{ }^{\circ} \mathrm{C}$ for $72 \mathrm{~h}$, which ensures full hydration according to the thermogravimetric data reported below. Labels like LBG10 are used in the following text, where 10 represents the barium excess percent, i.e., $\mathrm{La}_{0.9} \mathrm{Ba}_{1.1} \mathrm{GaO}_{4-\delta}$; in the case of dry powders, the letter " $\mathrm{D}$ " is attached to the acronym. Single-phase formation and purity was checked with a Bruker D500 laboratory diffractometer. The equilibrium constant of reaction 3 was measured by thermogravimetric analysis (TGA), using a Sartorius 7014 balance. The sample was first completely dehydrated in pure nitrogen at 900 ${ }^{\circ} \mathrm{C}$, then cooled very slowly (from 30 to $3 \mathrm{~K} / \mathrm{h}$ ) at constant water pressure $\left(P_{\mathrm{H}_{2} \mathrm{O}}=23 \mathrm{hPa}\right)$ to guarantee equilibration during the hydration. ${ }^{1}$ Diffuse-reflectance FT-IR (DRIFT) measurements were taken with a Bruker IFS 66 spectrometer using $\mathrm{KBr}$ as background. The IR spectrum of LBG20D was subtracted to LBG20 to isolate the contribution of protons to the IR signal.

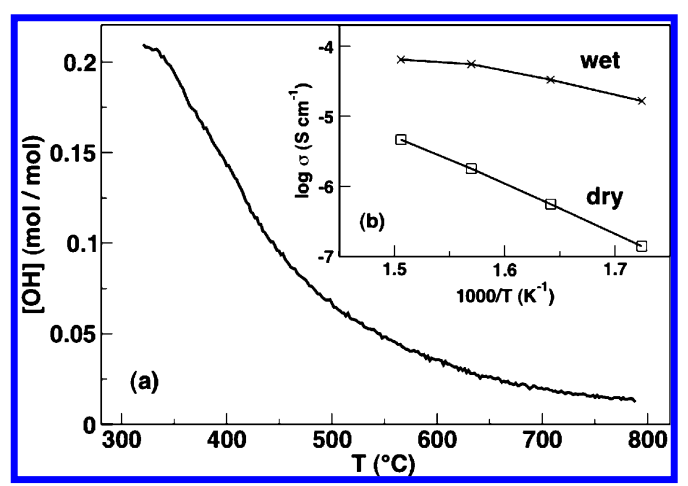

Figure 1. (a) Proton concentration in LBG20 as a function of temperature from TGA data. (b) Total conductivity of LBG20 in wet atmosphere and in dry atmosphere.

The calcined powders were isostatically pressed at $800 \mathrm{MPa}$. Attempts to sinter the compacted powders at $1500{ }^{\circ} \mathrm{C}$ resulted in melting and evaporation of LBG: therefore, a sintering temperature of $1350{ }^{\circ} \mathrm{C}$ was used, with $12 \mathrm{~h}$ of dwelling time, yielding a pellet density about $95 \%$ of the maximum. The surface of the pellets was then polished, and the electrodes were applied on each face by painting Pt paste and firing at $1000{ }^{\circ} \mathrm{C}$ for $12 \mathrm{~h}$. Conductivity of the pellets in both wet $\left(P_{\mathrm{H}_{2} \mathrm{O}}=23\right.$ $\mathrm{hPa}$ ) and dry $\mathrm{N}_{2}$ was measured with impedance spectroscopy from 5 to $10^{7} \mathrm{~Hz}$ using a Hewlett-Packard LCR Meter 4284 and modeled using two RQ equivalent circuits corresponding to bulk and grain boundary responses. At low temperatures, the bulk semicircle extended throughout the whole spectrum, so that only one contribution was used. In the following discussion, only bulk conductivity is considered.

XRD measurements in transmission geometry were collected in two experiments at the European Synchrotron Radiation Facility (ESRF, Grenoble). In situ XRD from 25 to $900{ }^{\circ} \mathrm{C}$ was taken at the ID11 beamline, with $\lambda=0.34 \AA$, in the range 0.3 $\leq q \leq 3.5 \AA^{-1}, q=4 \pi \sin (\theta) / \lambda$. The samples were mounted in a glass capillary connected to the gas flux inside a Linkam thermochemical cell, and the diffracted beam was collected using a 2-dimensional Frelon2K camera. High-resolution XRD was collected at the ID31 beamline, with $\lambda=0.4 \AA$, in the $q$-range $0.5-10 \AA^{-1}$. The instrumental contribution to the fwhm is about $0.003^{\circ}$. The sample was mounted in a spinning glass capillary and the diffracted radiation was collected by a bank of eight $\mathrm{Si}(111)$ analyzer crystals in front of scintillation detectors. The diffraction patterns were analyzed with GSAS, and the crystal and electronic structures were plotted with VESTA. ${ }^{17,18}$ Incident wavelength, zero displacement, and instrumental broadening were determined with $\mathrm{Si}$ and $\mathrm{LaB}_{6}$ standards and fixed in the fittings. The following parameters were fitted for each sample: lattice parameters, atomic positions, strain broadening, and isotropic thermal parameters. X-ray absorption spectra (XAS) on the Ga K-edge (10.4 keV) were collected in transmission mode at $80 \mathrm{~K}$ at the GILDA BM8 beamline of ESRF; data at room temperature were also collected at the XAFS beamline of Elettra (Trieste). The extended X-ray absorption fine structure (EXAFS) spectra were analyzed using Feff 8.4 and Viper. ${ }^{19,20}$

\section{Results and Discussion}

3.1. TGA and Conductivity. TGA and conductivity data for LBG20 are shown in Figure 1. The modeling of TGA data are equivalent to the case of perovskites described by Kreuer, ${ }^{21}$ modifying the equations according to the different oxygen stoichiometry as follows: 


$$
\begin{gathered}
{\left[\mathrm{O}_{\mathrm{O}}^{x}\right]+\left[\mathrm{V}_{\mathrm{O}}^{\bullet \bullet}\right]+\left[\mathrm{OH}_{\mathrm{O}}^{\bullet}\right]=4} \\
2 \cdot\left[\mathrm{V}_{\mathrm{O}}^{\bullet \bullet}\right]+\left[\mathrm{OH}_{\mathrm{O}}^{\bullet}\right]-\left[\mathrm{Ba}_{\mathrm{La}}^{\prime}\right]=0 \\
K_{\mathrm{eq}}=P_{\mathrm{H}_{2} \mathrm{O}}^{-1} \cdot\left[\mathrm{OH}_{\mathrm{O}}^{\bullet}\right]^{2}\left(\frac{\left[\mathrm{Ba}_{\mathrm{La}}^{\prime}\right]-\left[\mathrm{OH}_{\mathrm{O}}^{\bullet}\right]}{2}\right)^{-1} \times \\
\left(4-\frac{\left[\mathrm{Ba}_{\mathrm{La}}^{\prime}\right]+\left[\mathrm{OH}_{\mathrm{O}}^{\bullet}\right]}{2}\right)^{-1}
\end{gathered}
$$

From linear regression of $\log K_{\mathrm{eq}}$ vs $1 / T$, the thermochemical parameters are determined. For LBG20, they are $\Delta H^{0}=-90$ $\mathrm{kJ} / \mathrm{mol}$ and $\Delta S^{0}=-118 \mathrm{~J} / \mathrm{molK}$ (the fit is shown in the Supporting Information). The above equations assume that the electronic defects are negligible with respect to protons and oxygen vacancies, as previously reported for $\mathrm{LBG}^{7}$

The hydration enthalpy, which is directly related to the oxygen basicity, is moderate, so that about half of the vacancies are filled at $450{ }^{\circ} \mathrm{C} .{ }^{22}$ However, the significant hydration entropy indicates a remarkable degree of ordering between "gas phase" protons and $\mathrm{O}-\mathrm{H}$ bonds in the LBG20 lattice. As a consequence, at high temperatures, the equilibrium is shifted toward the vapor phase, and the hydration of the LBG lattice decreases rather steeply. Then, it is possible that the proton defects are trapped in definite configurations in the lattice, hindering their reorientation by preferential interactions in one or two directions. As is discussed below, this interpretation is corroborated by the stretching frequencies of the $\mathrm{O}-\mathrm{H}$ bonds and also finds a structural basis in the crystal structure analysis of the hydrated compounds. In the presence of water vapor, the conductivity is largely protonic, reaching $10^{-4} \mathrm{~S} \mathrm{~cm}^{-1}$ at $400{ }^{\circ} \mathrm{C}$. In dry atmosphere, the conductivity is definitely lower. As it is often the case with oxide proton conductors, the oxygen vacancy conductivity has a higher activation energy with respect to the protonic conductivity (1.1 vs $0.75 \mathrm{eV})$.

3.2. EXAFS. The results of the EXAFS analysis are reported in Tables 1 and 2. As an example of the fit range and quality, the EXAFS data and fit of LBG15 are shown in Figure 2. The Fourier-transformed data are plotted without corrections for the phase shift. The EXAFS data, weighed by $k^{2}$, were fitted in $R$-space up to about $4 \AA$ : owing to the rather low symmetry, there are many interatomic distances around $\mathrm{Ga}^{3+}$, so the coordination shells were grouped as follows in order to keep the number of free parameters to a minimum. With reference to Tables 1 and 2: (a) The Ga-O first shell is modeled as a single shell, with distance $R_{1}$ and disorder $\sigma^{2} \mathrm{O}$. (b) One $\mathrm{Ga}-\mathrm{O}$ intertetrahedral short distance is also included, lying at about 3 $\AA$ and sharing the same disorder factor as the first shell. As discussed below, this latter approximation holds at low temperatures only. (c) The Ga-M second shell covers a wide range of distances. To keep the number of fitting parameters to a minimum, it is split in three contributions, whose distances are $R_{\mathrm{M} 1}, R_{\mathrm{M} 2}$, and $R_{\mathrm{M} 2}$ and whose disorder factors are $\sigma_{\mathrm{M} 1}^{2}, \sigma_{\mathrm{M} 2}^{2}$, and $\sigma_{\mathrm{M} 3}^{2}$, respectively. It is worth noting that, unlike the case of perovskites, the local structure around $\mathrm{Ga}^{3+}$ does not present colinear configurations that give rise to appreciable multiple scattering contributions to the EXAFS signal: it was therefore possible to model the experimental data using single scattering signals only.

The coordination numbers were kept fixed, as documented in previous studies on defective crystalline oxides: in this case, the minimum $\mathrm{Ga}-\mathrm{O}$ first shell coordination number is expected to be 3.9 for LBG20D, instead of 4 , so that the difference is
TABLE 1: Structural Parameters around $\mathrm{Ga}^{3+}$ in Hydrated LBG Samples, Derived from the EXAFS Data Analysis ${ }^{a}$

\begin{tabular}{llccccc}
\hline & & LBG0 & LBG5 & LBG10 & LBG15 & LBG20 \\
\hline$R_{\mathrm{O} 1}$ & $4 \times \mathrm{Ga}-\mathrm{O}$ & 1.86 & 1.86 & 1.85 & 1.85 & 1.85 \\
$R_{\mathrm{O} 2}$ & $1 \times \mathrm{Ga}-\mathrm{O}$ & 3.01 & 3.01 & 3.01 & 3.02 & 3.02 \\
$\sigma_{\mathrm{O}}^{2}$ & & 4.3 & 4.3 & 4.3 & 4.3 & 4.8 \\
$R_{\mathrm{M} 1}$ & $2 \times \mathrm{Ga}-\mathrm{M} 1$ & 3.24 & 3.24 & 3.25 & 3.24 & 3.24 \\
$\sigma_{\mathrm{M} 1}^{2}$ & & 3.8 & 4.0 & 4.2 & 4.6 & 6.0 \\
$R_{\mathrm{M} 2}$ & $4 \times \mathrm{Ga}-\mathrm{M} 2$ & 3.41 & 3.41 & 3.42 & 3.42 & 3.42 \\
$\sigma_{\mathrm{M} 2}^{2}$ & & 6.8 & 7.0 & 7.0 & 7.7 & 11 \\
$R_{\mathrm{M} 3}$ & $1 \times \mathrm{Ga}-\mathrm{M} 3$ & 3.99 & 3.99 & 3.99 & 3.99 & 4.00 \\
$\sigma_{\mathrm{M} 3}^{2}$ & & 2.5 & 3.0 & 3.0 & 3.3 & 6.2
\end{tabular}

${ }^{a} M=\mathrm{La} / \mathrm{Ba}$. Distances in $\AA$, disorder factors in $10^{-3} \AA^{2}$. Uncertainty is on the last digit.

TABLE 2: Structural Parameters around $\mathrm{Ga}^{3+}$ in Dry LBG Samples, Derived from the EXAFS Data Analysis ${ }^{a}$

\begin{tabular}{llcccc}
\hline & & LBG5D & LBG10D & LBG15D & LBG20D \\
\hline$R_{\mathrm{O} 1}$ & $4 \times \mathrm{Ga}-\mathrm{O}$ & 1.85 & 1.85 & 1.85 & 1.85 \\
$R_{\mathrm{O} 2}$ & $1 \times \mathrm{Ga}-\mathrm{O}$ & 2.99 & 2.99 & 2.98 & 3.01 \\
$\sigma_{\mathrm{O}}^{2}$ & & 4.4 & 4.7 & 5.3 & 5.7 \\
$R_{\mathrm{M} 1}$ & $2 \times \mathrm{Ga}-\mathrm{M} 1$ & 3.24 & 3.24 & 3.23 & 3.23 \\
$\sigma_{\mathrm{M} 1}^{2}$ & & 3.6 & 3.9 & 5.2 & 6.0 \\
$R_{\mathrm{M} 2}$ & $4 \times \mathrm{Ga}-\mathrm{M} 2$ & 3.41 & 3.41 & 3.40 & 3.41 \\
$\sigma_{\mathrm{M} 2}^{2}$ & & 6.6 & 7.5 & 9.5 & 11 \\
$R_{\mathrm{M} 3}$ & $1 \times \mathrm{Ga}-\mathrm{M} 3$ & 3.98 & 3.99 & 3.99 & 4.00 \\
$\sigma_{\mathrm{M} 3}^{2}$ & & 3.0 & 4.5 & 6.4 & 6.2
\end{tabular}

${ }^{a} M=\mathrm{La} / \mathrm{Ba}$. Distances in $\AA$, disorder factors in $10^{-3} \AA^{2}$. Uncertainty is on the last digit.

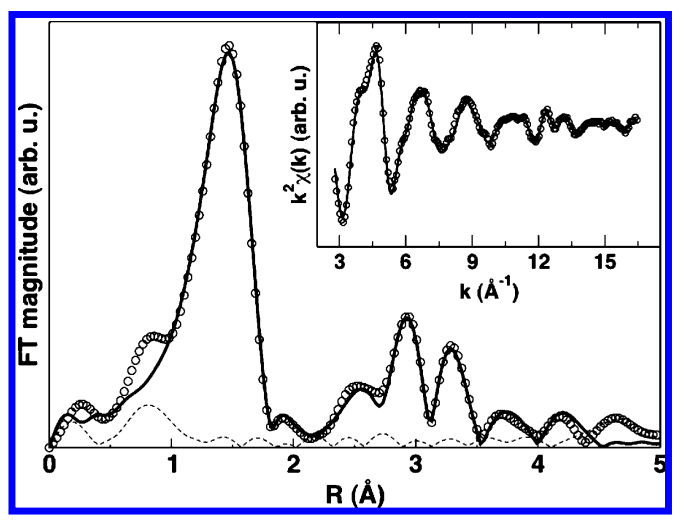

Figure 2. EXAFS data and model of LBG20 at $80 \mathrm{~K}$ : Fouriertransformed experimental data (circles), fit (line), and residual (dashes); in the inset, $k^{2}$-weighed EXAFS experimental data (circles) and fit (line).

lower than the uncertainty range. The cation defectivity can also be assumed to be largely lower than the EXAFS sensitivity. ${ }^{10-15}$

In $\mathrm{LBG}$ compounds, the average $\mathrm{Ga}-\mathrm{O}$ first-shell distance determined by EXAFS does not vary significantly on changing the self-doping and the hydration level. The disorder factor of the $\mathrm{Ga}-\mathrm{O}$ first shell, $\sigma_{\mathrm{O} 1}^{2}$, is $0.0043 \AA^{2}$ in LBG0 and reaches $0.0048 \AA^{2}$ in the LBG20 hydrated sample, with a relatively low increase for such a high self-doping level; the dry samples present an only slightly larger disorder, clearly due to the lattice perturbation produced by the insertion of oxygen vacancies. Apart from that, there are no significant structural differences between anhydrous and hydrated samples in the local environment of $\mathrm{Ga}^{3+}$. Regarding the formation of $\mathrm{Ga}_{2} \mathrm{O}_{7}$ clusters in dry LBG samples, as proposed by Kendrick et al., we have found no clear experimental evidence of the appearance of a $\mathrm{Ga}-\mathrm{Ga}$ short distance. ${ }^{8}$ The increase of the $\mathrm{Ga}-\mathrm{O}$ first shell disorder factors suggests that the $\mathrm{GaO}_{3}$ unit associated with a vacancy relaxes by displacing the oxygen atoms, rather than by forming 


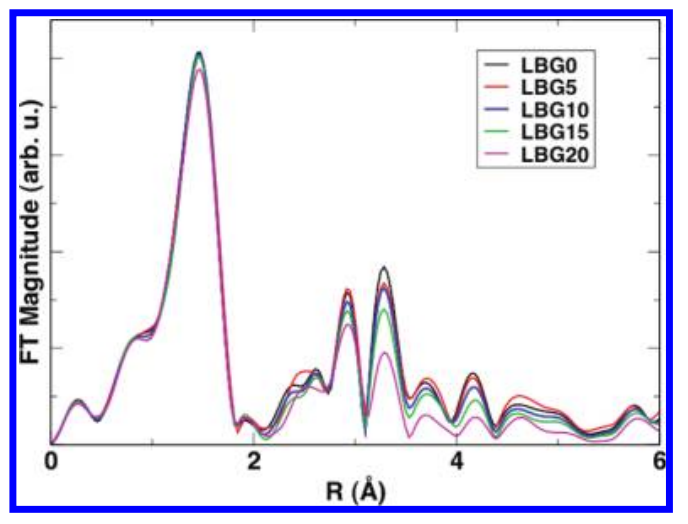

Figure 3. The $k^{2}$-weighed Fourier-transformed experimental EXAFS data of hydrated LBG samples at $80 \mathrm{~K}$. LBG0 is reported for reference.

a dimer with a neighboring $\mathrm{GaO}_{4}$ unit. It must also be kept in mind that the formation of transient $\mathrm{Ga}_{2} \mathrm{O}_{7}$ units was proposed during the migration of oxygen vacancies, so that it can also be that they are not seen in the time-averaged picture given by EXAFS. It can be concluded that, within the sensitivity of the EXAFS technique, the $\mathrm{GaO}_{4}$ tetrahedra respond like rigid bodies to the structural changes caused in the LBG matrix by $\mathrm{Ba} / \mathrm{La}$ substitution and proton insertion and removal.

In some proton-conducting perovskites, that is, In-doped barium zirconate ${ }^{14,15}$ and In-, Y-, and Gd-doped barium cerate, ${ }^{10-13}$ we observed a different behavior and in particular that the $\mathrm{Zr}-\mathrm{O}$ and $\mathrm{Ce}-\mathrm{O}$ first-shell correlations present a sharp increase of the Debye-Waller factors already at the lowest doping levels. The difference between gallates and perovskites is likely to reside in the respective network structure. Actually, in perovskites the $\mathrm{MO}_{6}$ octahedra share all corners, so that the structure and dynamics of each octahedron is tridimensionally coupled to the octahedra nearby, ${ }^{9}$ while in gallates the $\mathrm{GaO}_{4}$ units are not directly connected. As a consequence, self-doping has a large effect on the $\mathrm{Ga}-\mathrm{M}$ correlations $(\mathrm{M}=\mathrm{La}, \mathrm{Ba})$, mostly concerning the disorder factors $\sigma_{\mathrm{M}}^{2}$, that increase with increasing barium content, while the $\mathrm{GaO}_{4}$ tetrahedra are almost unaffected by the barium excess. Moreover, EXAFS does not detect large bond length variations of $\mathrm{Ga}$ with neighboring $\mathrm{M}$ atoms, demonstrating that the LBG structure is able to buffer the larger hindrance of $\mathrm{Ba}^{2+}$ and confirming the picture of rigid $\mathrm{GaO}_{4}$ units in a softer environment. The trends discussed above can be visually appreciated by inspection of Figure 3, showing that the self-doping level has little effect on the $\mathrm{Ga}-\mathrm{O}$ first coordination shell, while it has a noticeable influence on the amplitude of the further shells: the latter show little bond length variations, so the main effect of self-doping is on disorder in the mutual position of the $\mathrm{GaO}_{4}$ tetrahedral units and in the relative position of $\mathrm{GaO}_{4}$ with the spacer cations.

The temperature has very little effect on $\mathrm{Ga}-\mathrm{O}$ correlations, giving further evidence for the stiffness of the $\mathrm{GaO}_{4}$ tetrahedra. Figure 4 shows that between 80 and $300 \mathrm{~K}$, the disorder factor of first-shell $\mathrm{Ga}-\mathrm{O}$ in LBG0 does not vary, while the correlations between $\mathrm{Ga}^{3+}$ and the further shells are significantly smeared as an effect of thermal motions (the apparent contraction at $300 \mathrm{~K}$ results from the increased disorder and slightly anharmonic motion at higher temperatures). Apart from the $\mathrm{Ga}-\mathrm{M}$ peaks around $2.8-3.5 \AA$, a shallower but important effect can be observed of the rigid-body dynamics of the $\mathrm{GaO}_{4}$ tetrahedra on the peak at $2.5 \AA$, which corresponds to the intertetrahedral $\mathrm{Ga}-\mathrm{O}$ short distance. In fact, unlike the first shell peak, the $\mathrm{Ga}-\mathrm{O}$ distance linking two neighboring tetrahedra is perturbed by increasing temperature.

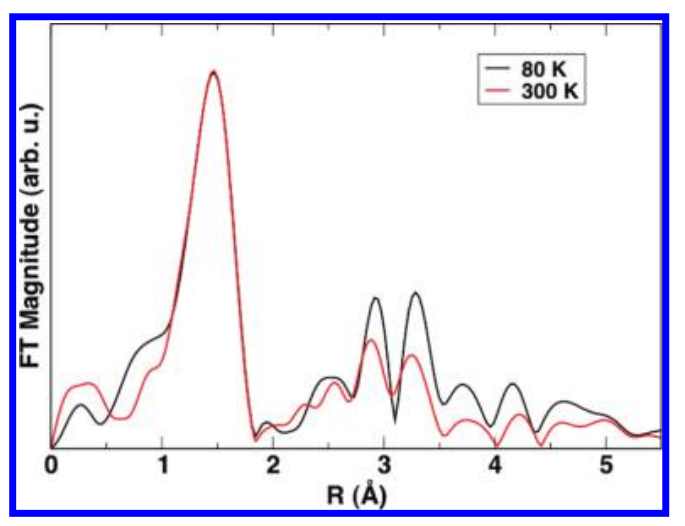

Figure 4. The $k^{2}$-weighed Fourier-transformed experimental EXAFS data of LBG0 at 80 and $300 \mathrm{~K}$.

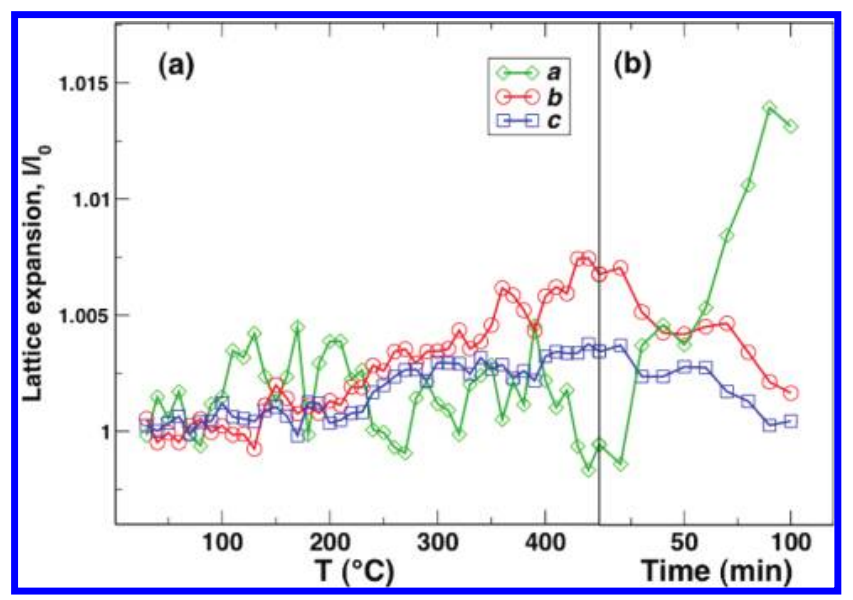

Figure 5. In situ $X R D$ of $L B G 20$ in dry $\mathrm{N}_{2}$ : lattice parameters, normalized for their value at room temperature. (a) Heating ramp from 25 to $450{ }^{\circ} \mathrm{C}$; (b) Drying at $450{ }^{\circ} \mathrm{C}$.

3.3. X-ray Diffraction. The LBG compounds do not show any phase transitions from room temperature to $900{ }^{\circ} \mathrm{C}$, so that in this interval the parent space group of LBG0, $P 2_{1} 2_{1} 2_{1}$, is retained by all of the samples. The average thermal expansion coefficient of LBG, as derived from in situ XRD, is $8 \times 10^{-6}$ $\mathrm{K}^{-1}$, comparable with that of $\mathrm{Y}: \mathrm{BaZrO}_{3} \cdot{ }^{1}$

Figure 5 shows the variation of the orthorhombic cell parameters of a hydrated LBG20 sample heated in dry $\mathrm{N}_{2}$ from 25 to $450{ }^{\circ} \mathrm{C}$ (left panel), and then held at constant temperature for about $2 \mathrm{~h}$ (right panel). The lattice parameters are normalized to the respective room temperature values. During the isothermal treatment, a remarkable expansion of the unit cell along the $a$ axis, and a less pronounced contraction along the two other edges takes place. Rehydration reversibly restores the starting structure and similar effects, although of less magnitude, are observed also for the lower doping levels. Then, the structural rearrangement is clearly related to the absorption/desorption of water in the crystal lattice of LBG and has been elucidated by the analysis of HR-XRD data.

The results of the Rietveld refinements are summarized in Table 3 and complete fitting parameters and fit quality factors are reported in the Supporting Information. Representative XRD data and fit of LBG20 are shown in Figure 6. It is worth noting that due to the very wide range of the diffraction data, and the high number of observed reflections, the structural parameters, especially as concerns the atomic coordinates, can be refined with high accuracy.

Despite the size mismatch between $\mathrm{La}^{3+}$ and the larger $\mathrm{Ba}^{2+}$ (about $0.3 \AA$ ), the $\mathrm{Ba}^{2+}$ excess in the self-doped LBG structure 
TABLE 3: Lattice Parameters and Isotropic Thermal Factors for the Dry and Hydrated Samples As Derived from Rietveld Refinement on HR-XRD ${ }^{a}$

\begin{tabular}{|c|c|c|c|c|c|}
\hline & LBG0 & LBG10 & LBG10D & LBG20 & LBG20D \\
\hline$a(\AA)$ & $10.0163(1)$ & $10.0402(1)$ & $10.0495(1)$ & $10.07522(8)$ & $10.1011(1)$ \\
\hline$b(\AA)$ & $7.2667(1)$ & $7.31288(4)$ & $7.29907(7)$ & $7.35619(7)$ & $7.31450(8)$ \\
\hline$c(\AA)$ & $5.91146(9)$ & $5.92460(3)$ & $5.92152(6)$ & $5.94627(4)$ & $5.93600(7)$ \\
\hline$V\left(\AA^{3}\right)$ & $430.268(4)$ & $435.001(4)$ & $434.355(4)$ & $440.709(4)$ & $438.578(4)$ \\
\hline$U_{\mathrm{Ga}}\left(10^{-3} \AA^{2}\right)$ & 2.5 & 5.9 & 8.2 & 10 & 15 \\
\hline$U_{\mathrm{O}}\left(10^{-3} \AA^{2}\right)$ & 1.9 & 8.4 & 11 & 18 & 32 \\
\hline$U_{\mathrm{La} / \mathrm{Ba}}\left(10^{-3} \AA^{2}\right)$ & 6.8 & 12 & 11 & 19 & 22 \\
\hline $\mathrm{Ga}-\mathrm{O}(\AA)$ & $1.846(1)$ & $1.822(2)$ & $1.830(2)$ & $1.819(4)$ & $1.824(5)$ \\
\hline
\end{tabular}

${ }^{a}$ Uncertainties are reported in parentheses, while uncertainty on the thermal factors is about $10 \%$.

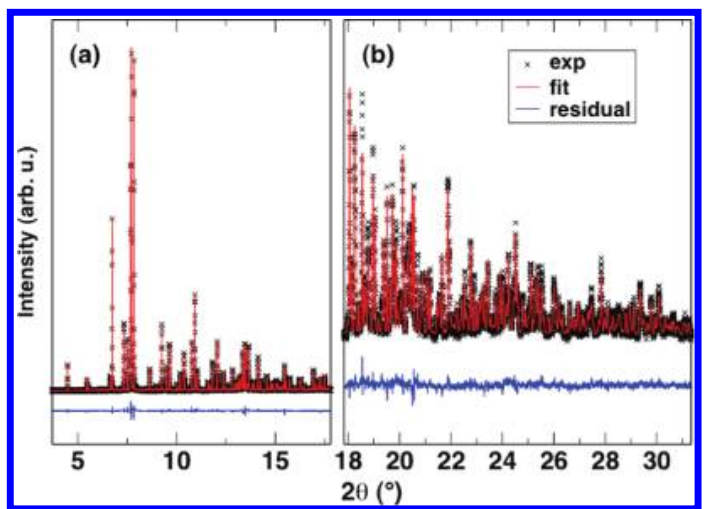

Figure 6. (a) HR-XRD pattern and Rietveld refinement of LBG20 $(\lambda$ $=0.4 \AA$ ). (a) Experimental data (black crosses), model (red line) and residual (blue line). (b) Zoom of the high-angle region.

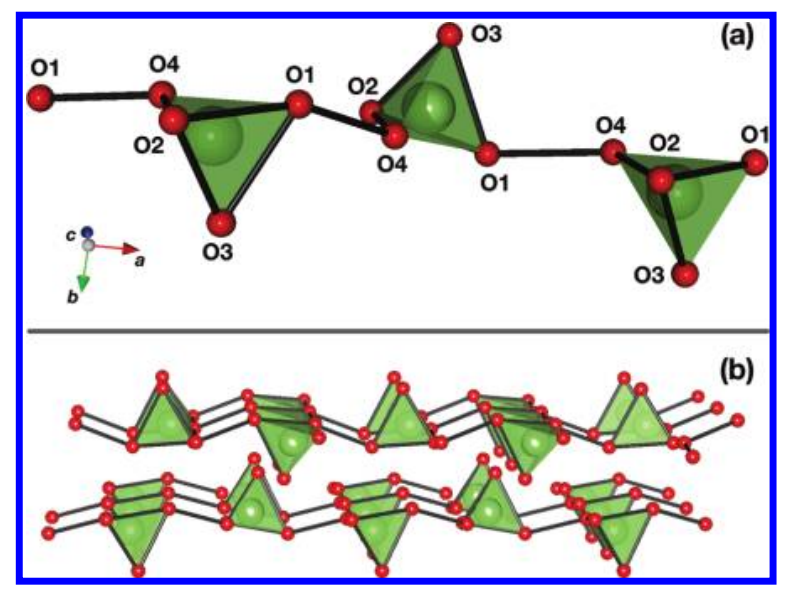

Figure 7. Fragment of the crystal structure of hydrated LBG20. The $\mathrm{La}$ and $\mathrm{Ba}$ atoms were removed for clarity. Only $\mathrm{O}-\mathrm{O}$ distances shorter than $3 \AA$ are shown as solid lines. (a) Three neighboring tetrahedra with labels for the oxygen atoms. (b) Network of tetrahedra connected through the $\mathrm{O} 1-\mathrm{O} 4$ paths.

is accommodated with minor rearrangements of unit cell size. These are best viewed by comparing the dry samples, to discriminate the effects of $\mathrm{Ba} / \mathrm{La}$ substitution from protonation: going from LBG0 to LBG20D (Table 3), the unit cell expands in the three directions, with the $a$ axis elongating more than the other two. On the contrary, when comparing dry and hydrated samples, an overall contraction is observed: the unit cell is squeezed along the $a$ axis and slightly expanded along $b$ and $c$. The refinement of atomic positions sheds light about the origin of this anisotropic rearrangement. A string of $\mathrm{O} 1-\mathrm{O} 4$ linked tetrahedra is drawn in Figure 7a: besides a slight reorientation of the $\mathrm{GaO}_{4}$ units, the hydration of LBG20D to form LBG20 produces an enlargement of the $\mathrm{Ga}-\mathrm{O} 1$ and of the $\mathrm{Ga}-\mathrm{O} 4$ intratetrahedral bond lengths, while the $\mathrm{O} 1-\mathrm{O} 4$ intertetrahedral distance is contracted by about $0.1 \AA$. The

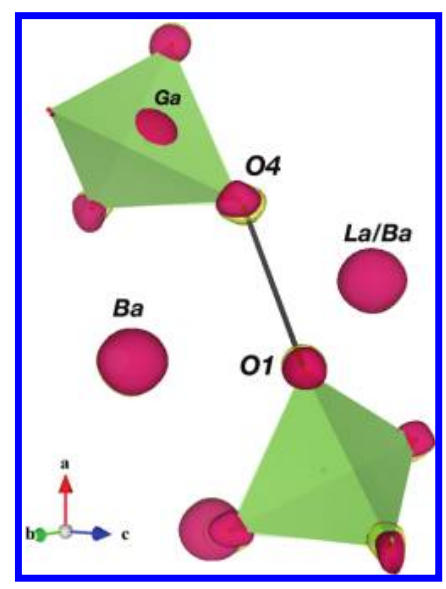

Figure 8. Experimental electron density map of LBG20 (yellow) and LBG20D (purple) calculated from HR-XRD data. The $\mathrm{GaO}_{4}$ groups are drawn as opaque solids, and the $\mathrm{O} 1-\mathrm{O} 4$ distance is evidenced for clarity.

overall shrinking of the $a$ axis is the resultant of these rearrangements. The other distances between neighboring tetrahedra remain nearly constant or even become slightly longer in the wet samples. A slab of the tridimensional network of LBG20 is reported in Figure 7b, showing that, among all of the $\mathrm{O}-\mathrm{O}$ distances between tetrahedra, only the $\mathrm{O} 1-\mathrm{O} 4$ distances are shorter than $3 \AA$.

Recently, computational modeling found that protons in LBG compounds form intertetrahedral hydrogen bonds. ${ }^{8}$ The analysis of HR-XRD data corroborates the theoretical prediction and in particular indicates that the most favorable site for the protons is in the $\mathrm{O} 1-\mathrm{O} 4$ interstitial region. In conclusion, the most relevant structural effect of hydration is that $\mathrm{O} 1$ and $\mathrm{O} 4$ are pulled together, likely due to the formation of an almost linear hydrogen bond. The formation of such bridges connecting neighboring tetrahedra provides a structural basis for the high hydration entropy determined by the above-reported analysis of thermogravimetry data, since such bridges provide a means for the hydroxyl defects to be well localized in the lattice.

Thanks to the wide reciprocal space range and to the high resolution of the experimental setup, very accurate experimental electronic densities can be obtained through the Fourier transform of the HR-XRD data. The 3D maps of LBG20 and LBG20D are superimposed in Figure 8, where the contraction of the $\mathrm{O} 1-\mathrm{O} 4$ distance can be appreciated: at the same time, the positions of the cations are unchanged.

As reported in Table 3, the isotropic thermal parameter of the anion sublattice, $U_{\mathrm{O}}$, increases by 1 order of magnitude when comparing LBG0 with LBG20. However, the EXAFS results point out that the $\mathrm{Ga}-\mathrm{O}$ first-neighbors bonds (and thus the $\mathrm{GaO}_{4}$ units) are substantially unperturbed by the barium excess. The discrepancy arises from the inherent peculiarities of the 


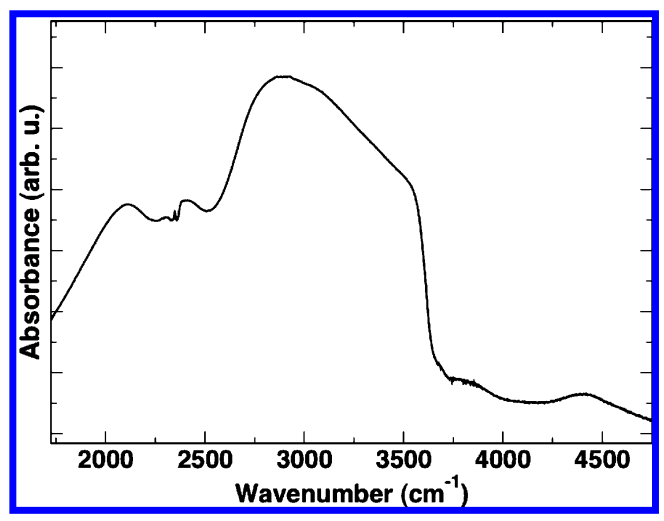

Figure 9. Infrared spectrum of $\mathrm{LBG} 20$ in the $\mathrm{O}-\mathrm{H}$ stretching region.

two techniques: through the Debye-Waller factor, EXAFS probes the distribution of interatomic distances from the absorbing species, while the XRD thermal factors are sensitive to the static and vibrational displacements of each atom with respect to its ideal lattice site. When defects are inserted in the lattice as a result of barium excess, the $\mathrm{GaO}_{4}$ units adopt different configurations throughout the lattice: in this way, the long-range order is disturbed, and the isotropic thermal factors increase sharply. Therefore, the local $\mathrm{Ga}-\mathrm{O}$ bonds are preserved, and at the same time new short intertetrahedral $\mathrm{O}-\mathrm{O}$ configurations are created. It is also interesting to note that the XRD thermal factors of gallium and oxygen decrease with hydration: the filling of oxygen vacancies is effective in reducing the distortion of the $\mathrm{GaO}_{4}$ units, but it is likely that also the establishment of intertetrahedral hydrogen bonds can have a role in restoring the order of the LBG network. Finally, the HRXRD data point out that the $\mathrm{GaO}_{4}$ units are not regular tetrahedra ( $\mathrm{Ga}-\mathrm{O}$ distances are detailed in the Supporting Information). This deformation is not resolved within the EXAFS accuracy, and is rather seen as an increase of the static disorder component of $\sigma^{2} \mathrm{O} 1$ (see Tables 1 and 2).

3.4. Infrared Spectroscopy and Proton Diffusion Mechanism. Infrared spectroscopy from 2000 to $4000 \mathrm{~cm}^{-1}$ probes the stretching motion of the $\mathrm{O}-\mathrm{H}$ bond. A classical review of structural and spectroscopic data of compounds with different degrees of hydrogen bonding established the relations between the degree of hydrogen bonding and the $\mathrm{O}-\mathrm{H}$ bond length, and between the latter and the stretching frequency: the $\mathrm{O}-\mathrm{H}$ stretching frequency increases with the $\mathrm{O}-\mathrm{H}$ bond strength, and decreases with the $\mathrm{O}-\mathrm{H}$.. .O hydrogen bond strength. Then, in an $\mathrm{O}-\mathrm{H} \cdots \mathrm{O}$ three-body arrangement, the stretching frequency depends on the separation between the two oxygen atoms: when the oxygens are too far apart (more than $3 \AA$ ), no hydrogen bond can be formed, and a "free" hydroxyl stretching frequency of about $3700 \mathrm{~cm}^{-1}$ is observed..$^{23}$ More recently, Karlsson and co-workers analyzed the $\mathrm{O}-\mathrm{H}$ IR spectrum of the proton conducting perovskite $\mathrm{In}: \mathrm{BaZrO}_{3}$, and proposed that different frequency components correspond to specific proton environments in the $2500-3700 \mathrm{~cm}^{-1}$ interval, with red-shifted frequencies corresponding to stronger hydrogen bond configurations. $^{24}$ The $\mathrm{O}-\mathrm{H}$ stretching region of the IR spectrum of LBG20 is shown in Figure 9. It is actually a group of bands extending from $2100 \mathrm{~cm}^{-1}$ to about $3500 \mathrm{~cm}^{-1}$, that includes contributions ranging from strongly hydrogen bonded configurations to loosely bound hydroxyls. By comparing the $\mathrm{O}-\mathrm{H}$ stretching bands of LBG20 with those of other protonconducting oxides, it can be observed that in LBG20 the modes corresponding to protons in strong hydrogen-bonded states are more populated. It is widely accepted that such states assist proton hopping, but hinder the reorientational freedom. ${ }^{25}$ From the LBG20 crystal structure refined from HR-XRD data, taking into account all the $\mathrm{O}-\mathrm{O}$ intertetrahedral separations, only the O1-O4 distance at $2.95 \AA$ can give rise to stable hydrogenbonded $\mathrm{O}-\mathrm{H}$ configurations. All of the other intertetrahedral distances, being larger than $3 \AA$, are too long for steady hydrogen bonding; nonetheless, transient bonds assisted by lattice vibrations can be formed, allowing proton hopping between the neighboring strings of $\mathrm{GaO}_{4}$ tetrahedra drawn in Figure $7 b{ }^{25}$ Actually, according to the theoretical forecast by Kendrick et al., intertetrahedral proton diffusion takes place with very low activation energy, and in particular barriers as low as $0.07 \mathrm{eV}$ are explicitly reported for the $\mathrm{O} 1-\mathrm{O} 2$ (with $3.14 \AA$ separation distance) and the $\mathrm{O} 3-\mathrm{O} 3$ (3.14 $\AA$ ) diffusion processes. $^{8}$

However, much higher activation energies were reported for intratetrahedral migration in the same work. The $\mathrm{GaO}_{4}$ tetrahedra are not regular, with entirely different $\mathrm{O}-\mathrm{O}$ distances ranging from 2.64 to $3.23 \AA$; moreover, the $\mathrm{Ga}^{3+}$ cation is shifted toward the $\mathrm{O} 1 \mathrm{O} 3 \mathrm{O} 4$ face and the structural environment of the $\mathrm{GaO}_{4}$ units is also highly anisotropic. As a consequence, it is not surprising that all of the intratetrahedral paths have different energy barriers. ${ }^{8}$ The stiffness of the $\mathrm{GaO}_{4}$ structure, hindering the facile formation of transient hydrogen bonds, is clearly at the origin of the much higher intratetrahedral activation energies. In conclusion, proton diffusion in LBG materials is biased by two detrimental factors: (i) compared to the case of perovskites, the proton local environment is clearly more asymmetric and therefore the proton diffusion is locally limited to preferential hopping directions; ${ }^{9}$ and (ii) while intertetrahedral hopping is straightforward, the stiffness of the $\mathrm{GaO}_{4}$ units hinders the intratetrahedral proton transfer.

\section{Conclusions}

We have investigated the structure and transport properties of LBG proton conductors with synchrotron radiation techniques (in situ XRD, HR-XRD, EXAFS), TGA, IR, and impedance spectroscopy. From the combined information obtained by these different techniques, it is possible to draw an overall picture of the relationship between proton conduction and structure of lanthanum barium gallates: (1) the $\mathrm{GaO}_{4}$ tetrahedra keep their substantially rigid shape after Ba self-doping and after hydration; (2) the structural rearrangements originated by hydration bring to a closer distance the $\mathrm{O} 1-\mathrm{O} 4$ couples of neighboring tetrahedra and involve an overall shortening of the $a$ orthorhombic axis; (3) it is therefore speculated that intertetrahedral $\mathrm{O}-\mathrm{H}$ bonds are stabilized by hydrogen bonding between the O1-O4 couples of adjacent tetrahedra; (4) the formation of a significant fraction of strong hydrogen bonds is reflected by the high hydration entropy measured with TGA and is confirmed by IR spectroscopy; (5) the theoretical prediction of low activation energy for intertetrahedral proton hopping can be related to the presence of strong hydrogen bonds that, however, probably limit proton reorientation; (6) the isotropy of migration paths of protons is also limited by the low symmetry of proton environment in LBG materials; (7) the stiffness of $\mathrm{GaO}_{4}$ tetrahedra, observed by EXAFS, can explain why the intratetrahedra activation barriers are much higher; (8) in the timeaveraged picture given by EXAFS, there is no clear evidence of $\mathrm{Ga}_{2} \mathrm{O}_{7}$ dimers in dry LBG compounds. The approach used for $\mathrm{LBG}$ can be exploited to investigate the structure-property relations of other proton-conducting oxides based on tetrahedral moieties, and to draw useful criteria for the development of new electrolytes. 
Acknowledgment. We acknowledge the ESRF and Elettra for provision of beamtime (Proposals CH-2714, MA-669 and 2008027). We thank Dr. Andrew N. Fitch, Dr. Aleksei Bytchkov (ESRF), and Dr. Luca Olivi (Elettra) for assistance during the XRD and EXAFS measurements, and Dr. Klaus-Dieter Kreuer (Max-Planck-Institut für Festkörperforschung, Stuttgart) for useful discussions. Partial financial support from Università di Palermo (ex 60\%) is acknowledged.

Supporting Information Available: Results and fit quality parameters of the Rietveld refinements; equilibrium constant of the hydration reaction for $\mathrm{LBG} 20$; impedance spectrum of LBG20 in wet atmosphere; XRD patterns for hydrated, dry, and rehydrated LBG20. This material is available free of charge via the Internet at http://pubs.acs.org.

\section{References and Notes}

(1) Kreuer, K.-D. Annu. Rev. Mater. Res. 2003, 33, 333.

(2) Slater, P. R.; Sansom, J. E. H.; Tolchard, J. R. Chem. Rec. 2004, $4,373$.

(3) Haugsrud, R.; Norby, T. Nat. Mater. 2006, 5, 193.

(4) Boysen, D. A.; Uda, T.; Chisholm, C. R. I.; Haile, S. M. Science 2004, 303, 68 .

(5) Karlsson, M.; Engberg, D.; Matic, A.; Panas, I.; Bowron, D. T.; Martin, S. W.; Nelson, C. R.; Martindale, C. A.; Hall, A.; Börjesson, L. Chem. Mater. 2008, 20, 6014

(6) Orera, A.; Slater, P. R. Chem. Mater. 2010, 22, 675.

(7) Li, S.; Schönberger, F.; Slater, P. Chem. Commun. 2003, 2694.
(8) Kendrick, E.; Kendrick, J.; Knight, K. S.; Islam, M. S.; Slater, P. R. Nat. Mater. 2007, 6, 871.

(9) Kreuer, K.-D. ChemPhysChem 2002, 3, 771.

(10) Longo, A.; Giannici, F.; Balerna, A.; Ingrao, C.; Deganello, F.; Martorana, A. Chem. Mater. 2006, 18, 5782.

(11) Giannici, F.; Longo, A.; Deganello, F.; Balerna, A.; Arico', A. S.; Martorana, A. Solid State Ionics 2007, 178, 587.

(12) Giannici, F.; Longo, A.; Balerna, A.; Kreuer, K.-D.; Martorana, A. Chem. Mater. 2007, 19, 5714.

(13) Giannici, F.; Longo, A.; Balerna, A.; Martorana, A. Chem. Mater. 2009, 21, 597.

(14) Giannici, F.; Longo, A.; Balerna, A.; Kreuer, K.-D.; Martorana, A. Chem. Mater. 2009, 21, 2641.

(15) Giannici, F.; Longo, A.; Kreuer, K.-D.; Balerna, A.; Martorana, A. Solid State Ionics 2010, 181, 122.

(16) Giannici, F.; Messana, D.; Longo, A.; Sciortino, L.; Martorana, A. J. Phvs.: Conf. Ser. 2009, 190, 012077.

(17) Larson, A.; Von Dreele, R. B. Report LAUR 1988, 86.

(18) Momma, K.; Izumi, F. J. Appl. Crvstallogr. 2008, 41, 653.

(19) Ankudinov, A. L.; Ravel, B.; Rehr, J. J.; Conradson, S. D. Phvs. Rev. B 1998, 58, 7565 .

(20) Klementev, K. D. J. Phys. D: Appl. Phvs. 2001, 34, 209.

(21) Kreuer, K.-D. Solid State Ionics 1999, 125, 285.

(22) Kreuer, K.-D.; Adams, S.; Münch, W.; Fuchs, A.; Klock, U.; Maier, J. Solid State Ionics 2001, 145, 295.

(23) Nakamoto, K.; Margoshes, M.; Rundle, R. E. J. Am. Chem. Soc. $1955,77,6480$.

(24) Karlsson, M.; Björketun, M. E.; Sundell, P. G.; Matic, A.; Wahnström, G.; Engberg, D.; Börjesson, L.; Ahmed, I.; Eriksson, S.; Berastegui, P. Phvs. Rev. B 2005, 72, 094303.

(25) Kreuer, K.-D.; Paddison, S. J.; Spohr, E.; Schuster, M. Chem. Rev. 2004, 104, 4637 .

JP1061896 